\title{
Percepção da Aids pelos profissionais da saúde que vivenciaram a epidemia durante o cuidado prestado às pessoas com a doença, em Florianópolis (SC), Brasil (1986-2006)
}

Perception of AIDS among health professionals who experienced the epidemic while caring for people with the disease in Florianópolis in the state of Santa Catarina, Brazil (1986-2006)

${ }^{1}$ Centro Administrativo, Centro de Saúde do Servidor, Secretaria de Estado da Administração de Santa Catarina. Rodovia SC $401 \mathrm{Km} 5 \mathrm{n}^{\circ} 4.600$, Saco Grande. 88.032-000 Florianópolis SC Brasil. nanyufsc@ibest.com.br ${ }^{2}$ Departamento de Enfermagem, Centro de Ciências da Saúde, Universidade Federal de Santa Catarina.
Abstract The scope of this study is to ascertain how the health professionals of a Reference Hospital for Infectious diseases experienced the AIDS epidemic while caring for people with the disease between 1986 and 2006. It involved a qualitative study with a socio-historical approach that used oral history to collect data from twenty-three health professionals. The following categories emerged from the content analysis: a change in profile of the AIDS epidemic; the improvement of care for people with HIV/AIDS; and an improvement in working conditions. The health professionals who experienced the epidemic while caring for patients with the disease perceived how much public health policies on AIDS, with advances and research, led to the consolidation of many laws. It also led to the coordination of health programs aimed at the improvement of services, assistance to people with the disease and ensuring a safer and less tiring work environment.

Key words Public health policies, Acquired immunodeficiency syndrome, Health care, Health professionals, Occupational health
Resumo O presente trabalho tem como objetivo conhecer como os profissionais da saúde de um Hospital Referência em doenças infectocontagiosas vivenciaram a epidemia da Aids, durante o cuidado prestado às pessoas com a doença, no período de 1986-2006. Estudo qualitativo com perspectiva sócio-histórica que utilizou a História Oral para coleta de dados com 23 trabalhadores da saúde. A partir da análise de Conteúdo emergiram tais categorias: Mudança do perfil da epidemia da Aids; Melhoria da assistência às pessoas com HIV/Aids; e, Melhoria das condições de trabalho. Os profissionais da saúde que vivenciaram a epidemia durante o cuidado às pessoas com a doença percebem o quanto que as políticas públicas acerca da Aids, com seus avanços e pesquisas, culminaram na consolidação de diversas leis, programas e coordenações de saúde, voltadas tanto à melhoria dos serviços, assistência às pessoas com a doença, quanto a conquista para um ambiente de trabalho mais seguro e menos desgastante.

Palavras-chave Políticas públicas de saúde, Sindrome da Imunodeficiência Adquirida, Assistência à saúde, Profissionais da saúde, Saúde do trabalhador 


\section{Introdução}

O surgimento da Aids e a percepção da sociedade, inclusive dos trabalhadores da saúde com relação a doença foi de medo, receio, preconceito. Pois até então, a Aids era notícia pela imprensa sensacionalista que semeava pânico na população, ao publicar imagens, matérias de caráter moralista e discriminatório. Como resposta a essa situação houve uma intensa mobilização da sociedade civil na estruturação de movimentos para reivindicação dos direitos aos pacientes, assim como de redefinição dos princípios pelos quais a sociedade abordava as pessoas infectadas pelo vírus. Com a evolução das respostas sociais e políticas em torno da doença, grandes foram as mudanças epidemiológicas ao longo dos primeiros 20 anos da Aids ${ }^{1}$

A evolução das políticas públicas de saúde com relação ao HIV/Aids aconteceu em fases distintas, considerando o desenvolvimento dos conhecimentos científicos com relação à doença, as articulações e a participação dos segmentos sociais e institucionais na formação das respostas à epidemia ${ }^{2}$.

Neste contexto é oportuno ressaltar ainda que as políticas públicas, a partir dos financiamentos disponibilizados aos serviços de HIV/Aids, proporcionaram uma melhoria na assistência às pessoas com a doença. Tais políticas e estudos culminaram na consolidação de diversas leis, programas e coordenações de saúde, assim como tiveram um preponderante papel na organização, na melhoria dos "Serviços de Assistência Especializada às pessoas com HIV/Aids", assim como nas práticas laborais dos trabalhadores da saúde 3 . Pois, em consequência ao surgimento da Aids, maior preocupação se teve com as políticas públicas voltadas à biossegurança dos trabalhadores da saúde, assim como com a estruturação e a organização dos serviços, que por sua vez contribuíram para a redução da sobrecarga de trabalho ${ }^{4}$.

Desta forma, acredita-se que as respostas sociais, assim como as políticas públicas em torno da Aids, foram de suma importância à mudança do perfil da epidemia, à qualidade da assistência às pessoas com HIV/Aids e à melhoria das condições de trabalho. Diante do contexto e ponderando acerca da temática exposta, decidiuse realizar este estudo que tem por objetivo: conhecer como os profissionais da saúde de um Hospital Referência em doenças infectocontagiosas vivenciaram a epidemia da Aids, durante o cuidado prestado às pessoas com a doença, no período de 1986-2006.
A opção de (re)construir a história deste período, 1986 à 2006, deve-se ao primeiro caso notificado de Aids em Florianópolis, município onde encontra-se localizado o Hospital Nereu Ramos (HNR), contexto deste estudo. E o recorte final até 2006, justifica-se pelo fechamento do Ambulatório de DST/Aids de Florianópolis, em virtude da descentralização deste serviço no município. O desejo de um estudo com profissionais da saúde, orientando-se pelas suas histórias e memórias, se coloca como importante por entender que as lembranças e as experiências, acerca das práticas de cuidado junto aos pacientes com HIV/Aids, ao longo da epidemia, revelam a importância e a influência dos avanços, das políticas públicas de saúde e de todo contexto político, econômico, social e histórico dessa epidemia, na evolução das práticas de cuidado.

\section{Metodologia}

Pesquisa sócio-histórica com abordagem qualitativa, que utilizou a História Oral (HO) como método-fonte para a coleta de dados. A história oral privilegia a realização de entrevistas com pessoas que presenciaram, testemunharam, participaram de acontecimentos num determinado contexto social ${ }^{5}$.

A HO, a ser utilizada como método-fonte, abriu espaço na história para aqueles que não tiveram voz sobre a sua própria história, no cuidado aos pacientes com HIV/Aids, ao longo da epidemia, através de suas memórias. Foram entrevistados, no período de março a outubro de 2011, quatro médicos, oito enfermeiras, quatro técnicos de enfermagem, três auxiliares de enfermagem, um dentista, um nutricionista, uma assistente social e uma psicóloga, totalizando 23 (vinte e três) profissionais da saúde que participaram, vivenciaram, direta ou indiretamente, do cuidado às pessoas com HIV/Aids, internadas no HNR, no período de 1986 à 2006. Os critérios de inclusão foram: trabalhadores da saúde que atuaram no cuidado aos pacientes com HIV/Aids, no período de estudo; que possuíam boa memória sobre o desenvolvimento das suas práticas laborais, no cuidado a essas pessoas; e que possuíam disponibilidade e interesse em participar da pesquisa.

A seleção dos sujeitos para participar da pesquisa foi realizada a partir de uma solicitação feita ao Setor de Recursos Humanos do HNR, e por recomendação dos próprios trabalhadores já entrevistados. Todas as entrevistas foram pre- 
viamente agendadas, conforme a disponibilidade do entrevistado, respeitando local, data e hora por eles sugeridas. As entrevistas ocorreram nos domicílios, e nos locais de trabalho dos sujeitos do estudo. A coleta de dados foi encerrada a partir da sua saturação.

Após o processo de coleta de dados, os mesmos foram transcritos, a fim de preservar a confiabilidade dos relatos. A transcrição e a organização dos relatos obtidos pela entrevista constituíram a ordenação dos dados e a classificação ocorreu a partir de exaustivas leituras e releituras, de modo a agrupar/compilar provisoriamente os possíveis enunciados. Nesse percurso, procurou-se identificar estruturas de relevância e realizar o reagrupamento por temas, conforme estabelece a análise de conteúdo de $\operatorname{Bardin}^{6}$, na qual emergiram as categorias: Mudança do perfil da epidemia da Aids; Melhoria da assistência às pessoas com HIV/Aids; e, Melhoria das condições de trabalho.

Ademais, o estudo foi submetido ao Comitê de Ética em Pesquisa com Seres Humanos (CEP$\mathrm{SH}$ ) da Universidade Federal de Santa Catarina e aprovado. Os sujeitos que aceitaram participar assinaram um Termo de Consentimento Livre e Esclarecido (TCLE). Para garantir o anonimato, os sujeitos do estudo foram identificados por letras referentes às categorias profissionais e por números, no intuito de seguir a ordem cronológica na qual atuaram no HNR (por exemplo, médico M1, enfermeiro E3, técnico de enfermagem TE2, auxiliar de enfermagem AE1, dentista D1, assistente social AS1).

\section{Resultados e discussão}

Neste momento, apresentaremos os resultados desta investigação a partir de agrupamentos discursivos, possibilitando ao leitor vislumbrar o percurso de análise dos dados na busca da compreensão da percepção dos profissionais da saúde com relação à epidemia, durante o cuidado às pessoas com HIV/Aids internadas no HNR, no período do estudo.

A grande maioria dos profissionais entrevistados é procedente do estado de SC; a faixa etária variou de 46 anos a 70 anos, com uma média de 58 anos, tendo atuado no cuidado direto ou indireto junto aos pacientes com HIV/Aids, no HNR por um período de 2 a 37 anos, com uma média de 19,5 anos.

\section{Mudança do perfil da epidemia da Aids}

A mudança do perfil epidemiológico da Aids foi percebida e tão logo mencionada pelos trabalhadores da saúde que vivenciaram o cuidado junto aos pacientes ao longo da epidemia. Inicialmente, a maioria das internações no HNR era caracterizada por pacientes homossexuais, Usuários de Drogas Injetáveis (UDI) e alguns casos por transfusão de sangue. Na época, tal população acometida pelo HIV, considerada como grupo de risco, foi fortemente estigmatizada por seus comportamentos, até então não aceitos pela sociedade. Fato que se pode observar a partir das seguintes falas:

Os pacientes com Aids eram jovens, usuários de drogas, a maioria homossexuais. A doença até foi chamada de peste gay e as pessoas eram muito estigmatizadas pela comunidade, pela mídia, porque além da Aids tinha toda uma questão social por atrás, de drogas, prostituição, homossexualismo. (AS1)

A família não queria que seu familiar fosse visto, porque na época, ter Aids era o mesmo que ter comportamento promíscuo. E a mídia foi um horror, os repórteres ficavam aos redores do hospital para noticiar a 'cara da Aids'. (TE4)

A Aids, por se tratar de uma doença incurável, para a qual não se conhecia sua real forma de transmissão e que se mostrava devastadora ao organismo infectado, foi marcada por medos, fantasias negativas, sentimentos de morte anunciada. Associada ainda a tais sentimentos, a história da Aids foi carreada por muita discriminação, rejeição, decorrente do fato de acometer sobretudo os homossexuais, UDI, profissionais do sexo, pessoas estas já estigmatizadas pela sociedade.

Ainda neste contexto, conforme retratam as falas, os meios de comunicação, de certa forma, tiveram um papel determinante na discriminação das pessoas com a doença. A disseminação da doença foi intensamente acompanhada pela mídia, que se caracterizou como veículo decisivo na difusão de informações. Se por um lado, divulgou os esforços da comunidade científica em informar mais sobre a doença, por outro, trouxe também muitas consequências desfavoráveis para sua compreensão, uma vez que reforçava no imaginário coletivo a concepção da doença como consequência de condutas socialmente reprováveis ${ }^{7,8}$.

A premissa de que a infecção limitava-se aos grupos de riscos solidificou na população não abarcada por esse rótulo, a falsa sensação de imunidade à doença. Por outro lado, os homossexuais e os UDI passaram, a partir de um traba- 
lho de conscientização, a se precaver, utilizando preservativo nas relações sexuais e não compartilhando agulhas contaminadas. Já os heterossexuais, por se considerarem invulneráveis à transmissão do HIV, expuseram-se a comportamentos de risco. Conforme demonstram as falas:

O grande público do Nereu eram os pacientes usuários de drogas e homossexuais, mas depois estes passaram a se proteger e aqueles que se achavam invulneráveis, como as mulheres casadas, passaram a não se cuidar e começaram a se infectar. (P1)

Por ser considerada câncer dos homossexuais, este pessoal passou a se cuidar e a Aids começou a ser transmitida entre mulheres casadas que não se cuidavam, não usavam preservativo com seu esposo, por achar, penso eu, que com elas não iria acontecer e também pela concepção machista do marido de usar a camisinha. (E3)

A cultura machista, o sentimento de invulnerabilidade à transmissão do HIV, assim como a fidelidade até então esperada nos relacionamentos, fez com que as mulheres não exigissem de seus parceiros o uso do preservativo. Situações estas preocupantes, que repercutiram na mudança do perfil epidemiológico, uma vez que aumentou o número de casos da transmissão do HIV entre os heterossexuais, sobretudo entre mulheres casadas.

Segundo dados do Ministério da Saúde, apesar dos casos de Aids entre homens superarem os de mulheres, a diferença esta cada vez menor. Ao longo dos anos, os dados confirmam a mudança do perfil, não é a toa que no ano de 2012, a categoria de exposição entre homens foi distribuída em heterossexual $(43,5 \%)$, seguida de homossexual (24,5\%), bissexual (7,7\%) e UDI (4\%). Já entre as mulheres, neste mesmo ano, observase como categoria de exposição a heterossexual $(86,8 \%)$, seguida da UDI $(2,3 \%)^{9}$.

Em Santa Catarina, a realidade epidemiológica acerca da Aids não é diferente. O Estado apresenta como principal categoria de exposição, a heterossexual $(61,9 \%)$, seguida de UDI $(20,9 \%)$ e Homossexual $(9,1 \%)$. O primeiro caso em mulheres ocorreu em 1987, desde então o número de casos em mulheres vem crescendo e a razão de masculinidade vem diminuindo a cada ano, passando da razão de 4,8 em 1987, para 1,6 no ano de $2011^{10}$.

E em Florianópolis, capital do Estado de SC, onde encontra-se localizado o HNR, os dados também intensificam a feminização da epidemia. De acordo com a Vigilância Epidemiológica do município, desde os primeiros casos de Aids notificados, no ano de 1986 até o ano de 2010, fo- ram registrados 4.240 casos, sendo 2.689 em homens, 1.343 em mulheres e 208 em crianças menores de 13 anos de idade. Mais recentemente, até outubro de 2011, o SINAN registrou 4.545 casos de Aids no município ${ }^{11}$. Com relação ao sexo, atualmente, ainda há mais casos de Aids entre os homens que entre mulheres, no entanto, essa diferença vem diminuindo ao longo da epidemia e o aumento proporcional do número de casos de Aids em mulheres pode ser observado pela razão de sexos, que em 1989 foi de 7,3 casos em homens para cada mulher e em 2009 foi de 1,5 homens para cada mulher, configurando-se o fenômeno da feminização da epidemia na cidade, seguindo as tendências nacionais ${ }^{11,12}$.

\section{Melhoria da assistência}

às pessoas com HIV/Aids

Nesta categoria, a percepção dos profissionais apontou para a importância das políticas públicas de saúde, das pesquisas, dos avanços na melhoria da assistência às pessoas com HIV/Aids, a partir da estruturação do HNR, da oferta da terapia antirretroviral, do acesso precoce ao diagnóstico, assim como dos direitos aos pacientes, conquistados com a criação das Organizações Não Governamentais (ONG), leis, portarias, comissões voltadas à organização dos serviços de assistência às pessoas com HIV/Aids.

Inicialmente, antes mesmo do surgimento da Aids, em virtude da internação de pacientes por tuberculose, o HNR já era um hospital esquecido pelo governo, discriminado pela sociedade e inclusive pelos profissionais da saúde que não queriam trabalhar em tal instituição. Porém, com o advento da Aids e por ser este um hospital referência nas doenças infectocontagiosas, passou a receber, a partir das políticas públicas voltadas à melhoria da assistência das pessoas com HIV/ Aids, incentivos, financiamentos para sua estruturação.

Com o surgimento da Aids, o hospital passou a não ser mais esquecido, porque com as politicas públicas começaram a vir incentivos do governo. [...] uma das alas do hospital, chamada DIP II começou a ser ativada, mas como eram muitos pacientes a serem atendidos, a estrutura não dava. Depois, no início da década de 90, em 1991, foi reformada a unidade de tuberculose masculina e criamos a Ala 5, que era uma unidade maior, um pavilhão grande, com banheiros, ficamos daí com 36 leitos. (E2)

Observa-se, a partir dos relatos, a necessidade de estruturação do HNR, para assistência de 
qualidade aos pacientes internados, debilitados pela doença. A epidemia da Aids propagou-se de forma crescente, passando a exigir a adoção de estratégias efetivas por parte dos órgãos competentes, a destacar a criação de serviços especializados nas instituições de saúde para o cuidado das pessoas com a doença.

Neste contexto, o HNR, no final da década de 1980 e início de 1990, investiu, respectivamente, na implantação de um Ambulatório, assim como no Hospital-Dia, ambos os serviços direcionados à assistência dos pacientes que não necessitavam de internação. $\mathrm{O}$ atendimento ambulatorial, no HNR foi iniciado em 1987, formado na época por uma equipe multidisciplinar composta por dois médicos, duas enfermeiras, uma psicóloga e duas assistentes sociais ${ }^{13}$.

No entanto, com a crescente demanda dos casos de Aids que chegavam, foi necessária a ampliação das instalações do Hospital, tanto na área de internação, como na área ambulatorial, assim como foram criadas novas formas de atendimento, como o Hospital-Dia. Desta forma, no ano de 1991, através de convênio realizado pela Secretaria de Estado da Saúde com o Banco do Brasil, foram ampliadas as instalações de ambulatório, construção de gabinete odontológico e, principalmente, a ampliação da unidade para internação dos pacientes com HIV/Aids, denominada Ala $\mathrm{V}^{13}$.

Com a demanda de casos de Aids chegando, a gente foi atrás de verba para estruturação do Nereu. Fomos até Brasília e conseguimos dinheiro com o Ministério da Saúde para aumentar os números de leitos e para construir um ambulatório maior. (M2)

Tivemos muitas mudanças com a Aids. Foi construído um ambulatório que ficava na parte da frente do hospital, mas a localização já atrapalhava a dinâmica do serviço. Daí fomos atrás de financiamento e no início dos anos 90 construímos um ambulatório maior, abrimos mais leitos na ala 5 e implantamos o Hospital-Dia. (E3)

Nota-se que as políticas públicas com relação à epidemia da Aids tiveram como foco prestar uma assistência de qualidade às pessoas com a doença. Neste sentido, além de algumas adaptações realizadas nas acomodações do próprio hospital para aumentar o quantitativo de leitos, pode-se observar nas falas, que na época, alguns profissionais de saúde buscaram financiamentos, a fim de melhorar a infraestrutura do HNR e consequentemente a assistência aos pacientes.

No contexto de implantação e estruturação de serviços voltados para a melhoria da assistên- cia às pessoas com HIV/Aids é oportuno ressaltar as negociações iniciadas na década de 1990, mais precisamente no ano de 1993, do governo brasileiro com o Banco Mundial para adquirir o empréstimo, denominado "Projeto AIDS". Tais investimentos permitiram a estruturação de uma rede assistencial em diversas regiões do país, a destacar os Serviços Ambulatoriais, Hospitaisdia (HD), Serviços de Assistência Domiciliar Terapêutica (ADT), hospitais convencionais, entre outros ${ }^{2,14}$.

Ainda com relação aos avanços, pesquisas e políticas públicas de saúde acerca da Aids, os sujeitos do estudo mencionaram a importância da oferta da terapia antirretroviral (ARV) para a melhoria da assistência às pessoas com HIV/Aids.

O acesso à terapia ARV deu-se em 1996, quando o Brasil adotou a Lei No 9.313, de 13 de novembro de $1996^{15}$, a qual dispôs sobre a distribuição gratuita de medicamentos para todas as pessoas acometidas pelo HIV/Aids, mesmo contra recomendações e advertências do Banco Mundial. Tal proposta terapêutica denominada de High Active Antiretroviral Therapy $y^{16}$ foi uma grande conquista, uma vez que proporcionou maior sobrevida aos pacientes HIV positivo.

$O$ ano de 96 foi o divisor de águas na terapia, porque antes as pessoas adoeciam e morriam. E a partir de 96, com os estudos, financiamentos do governo voltados à assistência dos pacientes vieram mais remédios e a associação destas drogas foi excelente, melhorou a sobrevida do paciente. A Aids passou a ser considerada doença crônica. (M2)

A disponibilidade da terapia antirretroviral de alta potência, gratuitamente, às pessoas acometidas pela doença causou impacto notável na realidade da epidemia, uma vez que reduziu a morbimortalidade, o quantitativo de internações hospitalares e aumentou a sobrevida dos pacientes, que passaram a viver mais e melhor. Estudo realizado pelo Departamento de DST, Aids e Hepatites Virais, demonstrou que a sobrevida de pessoas com diagnóstico entre 1995-1996 aumentou de mediana de 58 meses para 108 meses no período 1998-1999 ${ }^{17}$.

Neste cenário enfatizamos ainda que para a ampliação do acesso universal ao tratamento e sobrevida do paciente, fez-se necessário a priorização do acesso precoce ao diagnóstico do HIV e das infecções oportunistas. As políticas públicas em torno da Aids permitiram muitos financiamentos para a melhoria da assistência do paciente e, neste contexto, não só o acesso a terapia antirretroviral, mas também ao diagnóstico precoce, a partir de mais e melhores exames. Foram investi- 
mentos fundamentais para o início do tratamento e, por consequência, à sobrevida do paciente.

No que se refere aos direitos à melhoria da assistência aos pacientes com HIV/Aids, estes foram conquistados também mediante a criação de ONG, leis, portarias e programas voltados à organização dos serviços, tanto à assistência das pessoas com HIV/Aids quanto à prevenção.

É essa iniciativa que consolida o surgimento das primeiras organizações de base comunitária e redes nacionais, como, por exemplo, o surgimento da rede GAPA (Grupo de Apoio e Prevenção à Aids) em muitos estados, o Grupo pela Vida em São Paulo e a Associação Brasileira Interdisciplinar de Aids (ABIA) no Rio de Janeiro².

Em Santa Catarina, a rápida disseminação da Aids não foi diferente, pois segundo a Diretoria de Vigilância Epidemiológica do Estado, no ano de 1986 foram notificados oito casos e em 1987 dezessete, e tal progressão para o estado representava a instalação da epidemia em níveis alarmantes. Por este motivo, no ano de 1987, a partir dos movimentos sociais, das organizações comunitárias, dos profissionais de saúde, tradicionalmente "militantes" da saúde pública, assim como de uma opinião pública perplexa com relação à doença como "sentença de morte", foi implantada a Coordenação Estadual do Programa DST/ Aids vinculada à Diretoria de Assuntos Básicos de Saúde da Secretaria de Estado da Saúde. Esta teve como objetivo coordenar, planejar, executar e controlar os recursos indispensáveis ao controle da doença no estado, em conformidade com as orientações do Ministério da Saúde ${ }^{10,18}$.

Nesta mesma época, as respostas da sociedade civil frente a Aids foram sendo elaboradas no Estado, com a criação das primeiras ONG, como o GAPA, no ano de 1987, e a Fundação Açoriana para o Controle da Aids (FAÇA), em 1991 ${ }^{13}$.

A gente vivenciou a construção da epidemia. Lembro que fiz parte da fundação do GAPA e da FACA, e estas ONGs foram muito importante para as ações, políticas sociais, em defesa dos direitos humanos, trabalhistas dos pacientes com Aids. O GAPA teve um papel decisivo, de apoio para os pacientes com Aids. Eles brigavam por mais leitos, pegavam autorização judicial, iam atrás de medicamento. (E2)

Quando entrei já tinha o GAPA, criado por alguns dos funcionários do próprio hospital, mais voltado a parte da assistência, lutavam por mais leitos, medicamentos. Depois tivemos a FAÇA, eu inclusive participei da criação desta, que era mais voltada aos movimentos de lutas pelos direitos previdenciários, a não discriminação, de apoio. (M3)
O HNR esteve a frente das políticas públicas para a melhoria da assistência aos pacientes com HIV/Aids e neste cenário, ambas as ONGs, GAPA e FAÇA marcaram presença na história da Aids no estado de Santa Catarina, por terem sido as primeiras respostas da sociedade à epidemia.

As ONGs foram importantes na participação das reivindicações e nas lutas pelas conquistas que ainda necessitavam ocorrer junto aos órgãos governamentais e não governamentais e principalmente nas questões sociais que marcaram o início da epidemia no Estado. A destacar, a cobrança das autoridades por uma assistência de qualidade aos pacientes nos serviços públicos de saúde; a garantia do tratamento ARV gratuito; assim como uma política efetiva de controle do sangue e seus hemoderivados, notadamente a obrigatoriedade da implantação dos testes AntiHIV nos hemocentros ${ }^{13,18}$.

No que se refere à politização do sangue, no âmbito nacional, a Aids transfusional, ao disseminar grande medo, foi responsável pelos rumos da política nacional de sangue na década de 1980. Consequentemente, grandes foram os avanços conquistados com a pressão das ONG e de entidades da área da saúde, dado o grande número de casos de Aids registrados em decorrência da transfusão de sangue ${ }^{14}$.

Ainda no âmbito das políticas públicas na luta e controle da epidemia da Aids, sobretudo na assistência aos pacientes acometidos pela doença, o Ministério da Saúde criou em 1986 o Programa Nacional de DST e AIDS (PN-DST/AIDS), que em 2003 foi considerado como referência mundial, por diversas agências internacionais. $\mathrm{O}$ sucesso, inclusive mundial, de tal programa, dáse não só pela oferta universal e gratuita dos ARV, mas também por ser um programa interministerial em diálogo constante com os movimentos sociais e com a comunidade científica². Não é a toa que o Ministério da Saúde, ao longo da epidemia, tem veiculado junto à sociedade, sua preocupação com a organização dos serviços, voltados não só à assistência das pessoas com HIV/ Aids, mas também à prevenção e controle da epidemia.

No que diz respeito à organização dos serviços voltados à prevenção e controle da epidemia, neste estudo os sujeitos mencionaram a importância dada na época aos serviços de transfusão de sangue, na medida em que profissionais de diferentes instituições, reuniam-se para discutir a vigilância e o controle do sangue e seus hemoderivados. Fato que se pode evidenciar, a partir das falas: 
A situação dos serviços de sangue era caótica, a vigilância não atuava, não realizavam teste no sangue. [...] A gente começou a se organizar, criamos comissões para discutir sobre a organização do serviço de Aids no Estado. E uma das ações foi a implantação do serviço de sorologia para HIV, nos Hemocentros de todo Estado. [...] vivenciamos a construção da epidemia, foi um trabalho fantástico, anos lutando e tinha muita gente envolvida. (M2)

O advento da Aids e posteriormente o conhecimento de que a transfusão de sangue foi considerada um importante meio de transmissão do HIV, no início da epidemia, fez-se necessário organizar e realizar mudanças nos serviços de hemoterapia. Pois até então, a situação dos serviços de sangue era caótica, a Vigilância Sanitária não atuava nesta área de controle, logo, a maioria dos Centros de Hematologia do Estado não realizava nenhum teste no sangue ${ }^{13}$.

Assim, em 1987, foi criada a Comissão Interinstitucional para Controle da Aids, composta pelo antigo Departamento Autônomo de Saúde Pública (Diretoria da Vigilância Epidemiológica, Diretoria da Vigilância Sanitária, Laboratório Central de Saúde Pública); Centro de Hematologia e Hemoterapia de Santa Catarina (HEMOSC); Universidade Federal de Santa Catarina (UFSC); Instituto Nacional de Previdência Social (INAMPS); Fundação Hospitalar de Santa Catarina (FHSC). Esta comissão tinha por finalidade instituir uma política inicial para acompanhar, normatizar, estabelecer responsabilidades às instituições participantes da comissão e para acompanhar o desenvolvimento da epidemia em Santa Catarina. Ao Hemocentro, representado por um médico hematologista, competia criar a política de sangue no Estado ${ }^{13}$.

Nesse mesmo ano, a Comissão elaborou um diagnóstico estadual e constatou que somente os municípios de Florianópolis, Blumenau e Joinville realizavam os testes Anti-HIV. Assim, em 20 de julho de 1987, através do Decreto Lei Estadual no 272, foi criado o HEMOSC com o objetivo básico de prestar atendimento hemoterápico de qualidade à população da Grande Florianópolis, e assistir aos portadores de doenças hematológicas. E, através do Decreto Lei no 3015, de 27 de fevereiro de 1989 foi criado o sistema estadual de hematologia e hemoterapia com o objetivo de promover a interiorização das ações relativas ao uso de sangue, o qual obrigava a realização dos testes HIV em todo o Estado de SC. A partir desta data, o HEMOSC passou a realizar todos os testes de controle de qualidade do sangue no Estado ${ }^{10}$.
Contudo, ressaltamos que o engajamento vivenciado pelos profissionais da saúde, junto à sociedade civil em resposta à epidemia da Aids foi um exemplo de que as políticas públicas acerca da mesma tiveram um preponderante papel na melhoria dos serviços voltados tanto à assistência da pessoa com HIV/Aids, quanto à prevenção e ao controle da epidemia.

\section{Melhoria das condições de trabalho}

No que se refere à categoria melhoria das condições de trabalho, os sujeitos do estudo mencionaram a importância das políticas públicas, das pesquisas e dos avanços ao longo da epidemia da Aids, repercutindo significativamente em um trabalho mais seguro e menos desgastante no cuidado das pessoas com HIV/Aids.

A preocupação com o trabalho mais seguro, em consequência do surgimento da Aids, caracterizou-se pelas políticas públicas voltadas às medidas de biossegurança, a partir da implementação dos Equipamentos de Proteção Individual (EPI) (materiais descartáveis), da quimioprofilaxia após acidentes de trabalho, assim como pela implantação da vacinação contra Hepatite B. E, a subcategoria, trabalho menos desgastante, influenciando na melhoria de suas condições de trabalho, deuse pela contratação de mais funcionários, principalmente da enfermagem; diminuição da carga horária e acesso à Terapia Antirretroviral, a qual ampliou a sobrevida dos pacientes e passou a exigir menos dos trabalhadores da saúde.

O uso de materiais descartáveis, com destaque aos EPIs para a prática profissional segura, ratificou a importância das políticas públicas com seus financiamentos, investimentos voltados à biossegurança dos trabalhadores da saúde. Fato que podemos constatar, a partir dos relatos:

Muitas foram as melhorias para o paciente, mas também para a gente, principalmente para a enfermagem. Antes da Aids e no começo da epidemia, as seringa eram de vidro, as agulhas a gente reaproveitava e daí tínhamos que limpar, polir, não tinha fraldas descartáveis... tudo isso consumia mais e colocava a gente em risco. E com a Aids veio junto os financiamentos, a preocupação com a biossegurança, a chegada dos descartáveis para nossa segurança. (AE2)

Começaram a chegar as luvas descartáveis, coisa que antes da Aids não tinha. Nossos aventais e mascaras eram de pano, as seringas de vidro. E com o surgimento da Aids, maior e mais interesses, politicas públicas surgiram para a nossa segurança. (TE3) 
Nota-se que com o surgimento da Aids a maior preocupação foi dada pelas entidades governamentais, programas ministeriais para a proteção dos trabalhadores da saúde, no âmbito da sua prática laboral. Pois até então, antes do surgimento da doença e no início da mesma, não era preconizado o uso dos equipamentos de segurança, inclusive da luva, sendo esta, utilizada apenas nas cirúrgicas e para alguns procedimentos ${ }^{19}$.

Além dos EPIs, outras estratégias de biossegurança na prática profissional dos trabalhadores da saúde foram apontadas como determinantes para a melhoria das condições de trabalho, a destacar a conduta quimioprofilática após acidente de trabalho e a vacinaça contra a hepatite $B$. Os trabalhadores da saúde do HNR vivenciaram, ou melhor, usufruíram das políticas públicas em torno da Aids ao longo da epidemia, tendo em vista que trabalharam, como ainda alguns destes trabalham, em uma instituição considerada referência para o Estado de SC no atendimento às doenças infectocontagiosas. Assim, normas, protocolos ministeriais acerca da temática Aids e, sobretudo, a biossegurança dos trabalhadores foram rapidamente socializadas, no intuito de proporcionar melhores condições de trabalho.

A importância das políticas públicas na preconização do esquema completo para vacina contra hepatite B, assim como do protocolo de conduta pós-acidente de trabalho, foram medidas de biossegurança relevantes e necessárias, uma vez que minimizam a possível transmissão do HIV, entre outros patógenos veiculados pelo sangue, quando na eventual ocorrência do acidente de trabalho com exposição ao material biológico ${ }^{20,21}$.

Ainda com relação às políticas públicas influenciando na melhoria das condições de trabalho, o presente estudo abordou a subcategoria trabalho menos desgastante pelos trabalhadores da saúde, que os perceberam e neste sentido apontaram a importância dos movimentos e reivindicações realizados na época pela contratação de mais funcionários e diminuição da carga horária, e, por sua vez, menor sobrecarga. Conforme os relatos:

Ninguém queria trabalhar lá, lembro que só tinha eu de enfermeira, uns 5 ou 6 técnicos e o resto era tudo atendentes. Era muito puxado, não é a toa que eu pedia mais enfermeiros, até que em 1984, chegou enfermeiras e ficamos em três, mas depois uma delas foi embora. E com a Aids veio recursos, o hospital passou a ser lembrado, contrataram em 1988 mais enfermeiros, daí ficamos ao todo em nove. Puxa, a contratação de mais funcionários diminuiu a sobrecarga, melhorou nossas condições de trabalho. (E1)

Com o aumento de internação pela doença, houve a contratação de mais funcionários, inclusive eu entrei em 1988, com várias enfermeiras. $E$ as enfermeiras que entraram junto com as que estavam foi muito importante para a conquista de folgas, redução da carga horária, por melhores condições de trabalho. ( $\mathrm{P} 1)$

Percebe-se que com a demanda de pacientes com HIV/Aids, que aumentava a cada dia, além da ampliação de algumas instalações do hospital, também foi necessária a contratação de novos profissionais, principalmente da enfermagem para o atendimento das pessoas internadas pela doença.

Neste cenário, destaca-se a entrada das enfermeiras, em consequência do surgimento da Aids no HNR, e o preponderante papel que tiveram no sentido de resolver a complexa problemática para um cuidado seguro, buscando soluções em relação a material, equipamentos e, principalmente, organização do serviço de enfermagem para atuar com segurança junto aos pacientes com HIV/Aids ${ }^{22}$. Concordamos com as autoras e complementamos ainda o importante papel desempenhado pelas mesmas em conjunto com sua equipe e com as demais categorias profissionais na mobilização e nas reivindicações por melhores condições de trabalho, a partir de algumas transformações organizacionais, a destacar a diminuição da carga horária, a instituição de folgas nos aniversários, assim como o aumento da quantidade de profissionais para a enfermagem. Aproveitamos para ressaltar que por de trás destas lutas e reivindicações para a conquista de um trabalho menos desgastante e mais seguro aos trabalhadores da saúde no cuidado às pessoas com HIV/Aids, permearam também políticas públicas com seus financiamentos e investimentos voltados à assistência das pessoas acometidas pela doença.

Os avanços, as políticas públicas em torno da assistência às pessoas com HIV/Aids, a destacar o acesso aos ARVs, na segunda metade da década de 1990, como uma nova fase do tratamento, caracterizado por esquemas combinados e de alta potência, marcou a história da Aids ${ }^{17}$. Às pessoas com a doença, o acesso gratuito à terapia ARV aumentou a sobrevida e a qualidade de vida, e aos trabalhadores da saúde, sobretudo à enfermagem, a contratação de recursos humanos e a oferta dos ARV proporcionou um trabalho menos desgastante e de certa forma mais seguro. 


\section{Considerações finais}

As pesquisas, os avanços e as políticas públicas em torno da Aids, com seus financiamentos, até então vivenciados pelos profissionais da saúde durante o cuidado prestado às pessoas com a doença, no período do estudo, em muito contribuíram às percepções dos mesmos com relação à enfermidade.

Neste contexto, destaca-se a mudança do perfil da epidemia, a melhoria da assistência às pessoas com HIV/Aids, a partir da estruturação do HNR, do acesso à terapia antirretroviral, da melhoria aos meios de diagnóstico, assim como dos direitos aos pacientes, conquistados com a criação das ONGs, leis, portarias, comissões voltadas tanto à melhoria da assistência das pessoas com HIV/Aids, quanto à organização dos serviços de prevenção e controle da epidemia.
Ainda com relação às políticas públicas acerca da Aids, vale enfatizarmos a importância das mesmas para a melhoria das condições de trabalho dos sujeitos do estudo ao longo da epidemia, caracterizado pela preocupação das entidades governamentais com as estratégias de biossegurança dos trabalhadores, a ampliação dos serviços com a contratação de mais recursos humanos, assim como pelas lutas, reivindicações dos próprios profissionais da saúde, tradicionalmente militantes da saúde pública, por melhores condições de trabalho, enfim por um trabalho mais seguro e menos desgastante. Contudo, pensamos ser possível concluir que o HIV/Aids inaugurou uma nova forma de construir políticas públicas, pois apesar de ter provocado inúmeras mortes, no início da epidemia, a mesma ao ser reconhecida como um emergente problema de saúde pública, forçou a história da saúde a estabelecer diálogo com a sociedade e as políticas públicas.

\section{Colaboradores}

MV Villarinho foi a responsável pela concepção, delineamento, análise dos dados, redação do artigo e revisão crítica. MI Padilha colaborou na análise dos dados, na redação do artigo e na revisão crítica. 


\section{Referências}

1. Grangeiro A, Laurindo SL, Teixeira PR. Resposta à Aids no Brasil: contribuições dos movimentos sociais e da reforma sanitária. Rev Pan Salud Pública 2009; 26(1):87-94.

2. Souza BB, Vasconcelos CC, Tenório DM, Lucena MGA, Holanda RLT. A Política de AIDS no Brasil: uma abordagem histórica. J Manag Prim Health Care 2010; 1(1):23-26.

3. Villarinho MV. Evolução das práticas de cuidado dos trabalhadores da saude às pessoas com HIV/Aids em um hospital referência em doenças infectocontagiosas de Santa Catarina [tese]. Florianópolis: Universidade Federal de Santa Catarina; 2012.

4. Vieira M, Padilha MI, Pinheiro RDC. Análise dos acidentes com material biológico em trabalhadores da saúde Rev Latino-Am Enferm 2011; 19(2):332339.

5. Padilha MI, Borenstein MS, Bastiani J, Zytkuewisz $\mathrm{GV}$, Lessmann JC. As fontes historiográficas em pauta: a História Oral e a pesquisa documental. In: Borenstein MS, Padilha MI, organizadores. Enfermagem em Santa Catarina: Recorte de uma História (1900 - 2011). Santa Catarina: Secco; 2011. p. 37-58.

6. Bardin L. Análise de conteúdo. Lisboa: Edições 70 2004.

7. Monteiro S, Vilella WV, Knauth D. Discrimination, stigma, and AIDS: a review of academic literature produced in Brazil (2005-2010). Cad Saude Publica 2012; 28(1):170-176.

8. Marques Junior JS, Gomes R, Nascimento EF. Masculinidade hegemônica, vulnerabilidade e prevenção ao HIV/AIDS. Cien Saude Colet 2012; 17(2):511520.

9. Brasil. Ministério da Saúde (MS). Boletim Epidemiológico AIDS/DST, ano IX, n. 01, até semana epidemiológica 26 - junho de 2012. Brasília: MS; 2012. [acessado 2013 jan 14]. Disponível em: http://www. Aids.gov.br/sites/default/files/anexos/publicacao/ 2012/52654/boletim_jornalistas_pdf_22172.pdf

10. Santa Catarina. Secretaria de Estado da Saúde. Diretoria de Vigilância Epidemiológica. Gerência de Vigilância das DST/HIV/AIDS. 27 anos da epidemia de Aids: Uma História de Lutas, Avanços, Desafios e Conquistas; 2011. [acessado 2013 jan 7]. Disponível em http://www.dive.sc.gov.br/conteudos/ gerencia_dst_Aids/noticias/2011/Perfil_Aids_27 _anos_Epidemia.pdff

11. Florianópolis. Prefeitura Municipal. Secretaria Municipal da Saúde. Diretoria de Vigilância em Saúde. Gerência de Vigilância Epidemiológica. Aids em Florianópolis. Boletim informativo, 2011. [acessado 2012 nov 10]. Disponível em: http://www. pmf.sc.gov.br/arquivos/arquivos/pdf/05_12_2012_ 10.56.17.511309a742d7242fe1b2celaeela5f0e.pdf

12. Bastiani JAN, Padilha MICS, Vieira M, Maliska ICA, Maia ARCR. Pessoas que vivem com HIV/AIDS em Florianópolis/SC, Brasil: ocupação e status socioeconômico ocupacional (1986-2006). Rev. Eletr. Enf. 2012 [acessado 2013 jan 7];14(3):579-587. Disponível em: http://www.fen.ufg.br/revista/v14/n3/v14n3 a14.htm
13. Miranda AFB. História da AIDS em Santa Catarina: características de uma epidemia [dissertação]. Florianópolis: Universidade Federal de Santa Catarina; 2003.

14. Szwarcwald CL, Castilho EA. A epidemia de HIV/ AIDS no Brasil: três décadas. Cad Saúde Pública 2011; 27(Supl. 1):4-5.

15. Brasil. Lei No 9.313, de 13 de novembro de 1996. Dispõe sobre a distribuição gratuita de medicamentos aos portadores do HIV e doentes de AIDS. Diário Oficial da União 1996; 14 nov.

16. Couzigou C, Semaille C, Strat YL, Pinget R, Pillonel J, Lot F. Differential improvement in survival among patients with AIDS after the introduction of High Active Antiretroviral Therapy (HAART). AIDS Care 2007; 19(4):523-531.

17. Geocze L, Mucci S, Marco MAD, Nogueira-Martins LA, Citero VA. Qualidade de vida e adesão ao tratamento anti-retroviral de pacientes portadores de HIV. Rev Saude Publica 2010; 44(4):743-749.

18. Maliska ICA, Padilha MI, Meirelles BHS, Koller EMP, Andrade SR. A AIDS em Santa Catarina/Brasil: um histórico dos 25 anos da epidemia. Cad Saude Coletiva 2011; 19(2):147-156.

19. Gir E, Prado MA, Canini SRMS, Hayashida M. O impacto da Aids na prática de enfermagem: um problema de saúde pública. DST-J Bras Doenças Sex Transm 2005; 17(1):39-43.

20. Osti C, Machado JM. Vírus da hepatite B: avaliação da resposta sorológica à vacina em funcionários de limpeza de hospital-escola. Cien Saude Colet 2010; 15(Supl. 1):1343-1348.

21. Oliveira AC, Gonçalves JA. Acidentes com material biológico entre os profissionais de saúde: uma análise da cobertura vacinal para hepatite B no cenário brasileiro. Journal of Nursing UFPE 2007; 1(1):82-87. 22. Borenstein MS, Maia ARC, Vieira M, Padilha MI. Hospital Nereu Ramos de Florianópolis/SC: o poder das enfermeiras e as transformações a partir de seus saberes (1977-1986). In: Borenstein MS, Padilha MI, organizadores. Enfermagem em Santa Catarina: recorte de uma história (1900-2011). Florianópolis: Secco; 2011. p. 97-113.

Artigo apresentado em 30/04/2013

Aprovado em 26/05/2013

Versão final apresentada em 30/05/2013 